\title{
Terapia broncodilatadora ¿Qué responsabilidad tenemos los médicos y los pacientes en su uso inadecuado?
}

\section{Bronchodilator therapy}

\author{
What responsibility do we doctors and patients have in their misuse?
}

Las enfermedades pulmonares con compromiso broncoobstructivo incluyen una variedad de patologías que van desde la enfermedad pulmonar obstructiva crónica (EPOC) y el asma, pero también incluye otros trastornos como las enfermedades intersticiales dentro de las que se encuentran la bronquiolitis respiratoria, la fibrosis quística o las enfermedades vasculíticas con compromiso pulmonar como la poliangeítis granulomatosa eosinofílica (síndrome de Churg Strauss), causas infecciosas como las bronquiolitis virales, tuberculosis, neumonía y aspergillosis broncopulmonar alérgica y otras causas como neumonitis por broncoaspiración o cuerpos extraños en la vía aérea entre otras.

El mayor uso de inhaladores se concentra en la EPOC y asma por su mayor incidencia y prevalencia, lo cual genera un alto costo al sistema de salud para su manejo. La EPOC se estima que para el año 2030 será la cuarta causa de muerte (1) teniendo una carga económica actualmente en Europa de 38.6 mil millones de euros y en Estados Unidos sus costos directos se estiman en $29.5 \mathrm{mil}$ millones de dólares y los costos indirectos en 20.4 mil millones de dólares (2); el asma está presente en 235 millones de personas en el mundo (3) con un costo económico en Estados Unidos de 56 mil millones de dólares en 2007 o 3259 dólares por persona por año (en 2009), en Europa en el año 2011 se estimó que el costo total por año es de 19.3 mil millones de euros en personas entre 15 y 65 años (4). La prevalencia de la EPOC en Latinoamérica y el Caribe es de $13.4 \%$, encontrando en Colombia una prevalencia de 7.7\% (6.2-9.5, IC $95 \%$ ) que se incrementa con la edad en mayores de 50 años (5), mientras que el asma en Latinoamérica tiene una prevalencia total entre $6.9 \%$ (México) y $33.1 \%$ (Perú) (6) y la prevalencia en adultos en Colombia está estimada en $6.3 \%$ (7).

Teniendo en cuenta esta información donde un porcentaje alto de la población está o estará afectada por estas patologías es de vital importancia hacer un diagnóstico acertado y posteriormente proponer una terapia adecuada personalizada ajustada a las recomendaciones nacionales e internacionales, para optimizar los recursos para su diagnóstico y tratamiento, así como obtener el mejor control de estas enfermedades (2, 8-13).

El artículo de Machado JE et al (14) pone de manifiesto una de las dificultades al enfrentarse al tratamiento de estas patologías, no sólo presentes en países en desarrollo, sino también en los países desarrollados, como lo es la formulación inadecuada de la terapia inhalada, la cual tiene origen en múltiples factores como son un mal diagnóstico de la enfermedad y por ende una pobre respuesta al tratamiento, la sobredosificación del medicamento, el uso inadecuado de los dispositivos, falta de educación al paciente y del personal de salud y la verificación de su uso correcto. Todas estas variables nos llevan a un inadecuado control de estas patologías con exacerbaciones y hospitalizaciones frecuentes con el consiguiente aumento de la carga económica al sistema de salud que enfrenta un déficit fiscal importante a la fecha en nuestro país.

Se ha estimado el costo económico por el mal uso de los inhaladores, con base en el reporte de ventas de 500 millones de unidades al año y asumiendo un costo promedio de 50 dólares por inhalador, el gasto total sería de 25 mil millones de dólares anuales; teniendo en cuenta que se estima que el 28$68 \%$ de pacientes no usan el inhalador de la forma adecuada para obtener los beneficios del medicamento prescrito y que el $39-67 \%$ del personal de salud (enfermeras, médicos, terapeutas respiratorios) desconoce el uso correcto de los dispositivos para inhalación, los costos se incrementan por cada inhalador inadecuadamente formulado llevando a sobrecostos al sistema de salud entre 7 y 15.7 mil millones de dólares y adicional al factor
Ver artículo: página 218

Dr. Julio César Forero Forero: Internista Neumólogo, Msc Enfermedades Vasculares Pulmonares. Neumólogo, Hospital Pablo Tobón Uribe, Hospital La María, Neumovital. Medellín (Colombia).

E-mail: julcefo@yahoo.com 
económico se debe evaluar el impacto sobre el paciente quien cree que recibe un cuidado adecuado en salud, pero que continua padeciendo los síntomas evitables de su enfermedad y la morbimortalidad por su patología pobremente controlada. Adicionalmente el médico siente frustración al manejar estos pacientes, ya que no presentan una mejoría de su estado de salud y ante esta situación decide el escalamiento del tratamiento en un intento por mejorar al paciente llevando a incremento en los costos por medicamentos (15). La solución es la educación del paciente y del proveedor del cuidado en salud, pero en el esquema de atención actual no existe la posibilidad de dedicar el tiempo suficiente para la educación del paciente en consulta, si se gastaran 30 dólares al año por cada usuario de inhaladores, esto representaría una inversión de 900 millones de dólares pero llevaría a un ahorro al sistema de salud de más de cinco veces el costo de la inversión (15).

A nivel local se han realizado algunos estudios al respecto, como el llevado a cabo en la ciudad de Bogotá (16) en un hospital de tercer nivel de atención, donde en forma prospectiva se aplicó una encuesta a los pacientes hospitalizados encontrando como resultado grandes deficiencias en factores educacionales y estrategias insuficientes en el entrenamiento de los pacientes para el uso de inhaladores, adicionalmente se encontraron diferencias relacionadas en el nivel de educación, estrato socioeconómico, mayor edad y ocupación actual, asociándose a una peor técnica de inhalación un nivel socioeconómico bajo, edad adulta mayor y desempleo.

Existen tres tipos clásicos de no adherencia a la terapia: el subuso, el sobreuso y el uso inapropiado (17). El subuso es la reducción de la dosis diaria con respecto a la dosis estándar de medicación. El uso inapropiado es confirmado por la determinación de que la medicación es inefectiva, no indicada o si hay una duplicación innecesaria de la terapia, lo cual se relaciona con factores como ser mayor de 65 años con polifarmacia (toma de dos o más medicamentos), raza blanca, ingresos por menos de 30000 dólares al año, más de 6.8 medicamentos prescritos y baja percepción de mejoría por el paciente. En la población estudiada por Machado et al, son pacientes mayores de 65 años (promedio de edad 73 años) con polifarmacia por las comorbilidades en la muestra estudiada, lo que sugiere que esta población tiene problemas de adherencia al tratamiento llevando a consultas más frecuentes y llevando al médico a una sobre dosificación de inhaladores del mismo grupo farmacológico para lograr la mejoría del estado de salud del paciente. Esta hipótesis requiere su confirmación con la realización de estudios clínicos en Colombia.

De igual importancia es la educación a los médicos, en una encuesta a 1500 médicos, sólo $14 \%$ tenía conocimiento adecuado de la terapia inhalada y de éstos, menos de 50\% conocía la maniobra inhalatoria correcta con inhaladores de dosis medida y solamente $25 \%$ revisaba la técnica inhalatoria del paciente antes de prescribir un nuevo inhalador o medicación combinada (18).

Se pueden realizar varias estrategias de entrenamiento como visitas a los profesionales del cuidado de salud usando demostraciones con placebo o el propio inhalador del paciente, videos de demostración de técnicas de uso adecuado e incorrecto, teleasesoría y programas basados en la web. Todos los profesionales de la salud deben tener el entrenamiento: médicos de cuidado primario, neumólogos, enfermeras, fisioterapeutas y farmacéuticos (19).

Se requieren estudios en Colombia para determinar la adherencia por parte del personal médico a las guías internacionales, definir el conocimiento de los inhaladores y dispositivos disponibles en el mercado por parte del personal de salud y realizar campañas de educación para evitar la sobreformulación de los inhaladores. Adicionalmente se debe educar al paciente acerca de su enfermedad pulmonar, el uso de los dispositivos de inhalación y el efecto de los inhaladores sobre su enfermedad, deben realizarse programas de seguimiento para evaluar el uso de inhaladores en el tiempo.

Se debe insistir a las autoridades de salud, con la evidencia disponible que apoya el uso de broncodilatadores de larga acción solos o en combinación con corticoides inhalados, la inclusión en el Plan Obligatorio de Salud (POS) de las nuevas moléculas solas y/o en combinación, dado que las actualmente disponibles no son la terapia de elección en la mayoría de los casos para el manejo de las patologías respiratorias y si están contribuyendo con aumentar el déficit fiscal por su mal uso, la sobreformulación y mal control de las enfermedades con exacerbaciones frecuentes 
y hospitalizaciones evitables que también impactan la calidad de vida de la población y el bienestar del paciente y su familia.

\section{Referencias}

1. Mathers CD, Loncar D. Projections of Global Mortality and Burden of Disease from 2002 to 2030. PLoS Med 2006; 3(11): e442. doi: 10.1371/journal.pmed.0030442.

2. Global Initiative for Chronic Obstructive Lung Disease. Global strategy for the diagnosis, management, and prevention of chronic obstructive pulmonary disease Updated 2015.2015. Acceso: Septiembre 2015. Hallado en: http://www.goldcopd. org/uploads/users/files/GOLD_Report_2015_Sept2.pdf

3. WHO. Asthma. Secondary Asthma. Update November 2013. Acceso: septiembre 2015. Hallado en: http://www.who.int/ mediacentre/factsheets/fs307/en/

4. The Global Asthma Report 2014. Economic burden of asthma. Acceso septiembre 2015. Hallado en: http://www. globalasthmareport.org/burden/economic.php.

5. Ciapponi A, Alison L, Agustina M, et al. The epidemiology and burden of COPD in Latin America and the Caribbean: systematic review and meta-analysis. COPD 2014; 11: 339350.

6. Forno E, Mudita G, Cepeda A, et al. Asthma in Latin America. Thorax 2015; 70: 898-905.

7. Dennis R, Caraballo L, Garcia E, et al. Prevalence of asthma and other allergic conditions in Colombia 2009-2010: a cross-sectional study. BMC Pulmonary Medicine 2012; 12: 17.

8. Global Initiative for Asthma. Global Strategy for Asthma Management and Prevention, 2015. Available from: www. ginasthma.org.

9. NICE. Chronic obstructive pulmonary disease. NICE clinical guideline 101.2010. Available from: www.guidance.nice.org. $\mathrm{uk} / \mathrm{cg} 101$.

10. SIGN. British guideline on the management of asthma. A national clincal guideline. 2014. Available: www.brit-thoracic. org.uk/document-library/clinical-information/asthma/btssignasthma-guideline-2014/

11. Miravitlles M, et al. Guia española de la EPOC (GesEPOC). Actualización 2014. Arch Bronconeumol 2014; 50 (Supl 1): 1-16.

12. GEMA. Guía española para el manejo del asma. 2015. Disponible en: www.gemasma.com.

13. Londoño D, García OM, Celis C, et al. Guía de práctica clínica basada en la evidencia para la prevención, diagnóstico, tratamiento y seguimiento de la enfermedad pulmonar obstructiva crónica (EPOC) en población adulta. Acta Med Colomb 2014; 39 (Supl. 3): 1- 48.

14. Machado JE, Moreno PA, Bañol AM. Patrones de prescripción de broncodilatadores y corticoides inhalados en pacientes adultos de Colombia. Acta Med Colomb 2015; 218-26.

15. Fink JB, Rubin BK. Problems with inhaler use: a call for improved clinician and patient education. Respir Care 2005; 50: $1360-1374$.

16. Vargas O, Martínez J, M Ibanez, et al. The use of metereddose inhalers in hospital environments. J Aerosol Med 2013; 26:287-96.

17. Restrepo RD,Alvarez, MT, Wittnebel LD, et al. Medication adherence issues in patients treated for COPD. Int $J$ COPD 2008; 3:371-384.

18. Plaza V, Sanchis J, Roura P, Molina J, Calle M, Quirce S, et al. Physicians' knowledge of inhaler devices and inhalation techniques remains poor in Spain. J Aerosol Med Pulm Drug Deliv 2012; 25: 16-22.

19. Roche N, Chrystyn H, Lavorini F, et al. Effectiveness of inhaler devices in adult asthma an COPD. EMJ Respir 2013; 1: 64-71. 